\title{
Initial Thermal Response of Micro/Nano-Al particle Embedded in Ener- getic Materials Excited by Single-Pulse Laser
}

\author{
Yajing Peng ${ }^{* * *}$, Jianing Meng ${ }^{*}$, Xing Fu ${ }^{*}$ and Yanxue Jiang ${ }^{*}$ \\ * Department of Physics, Bohai University, Jinzhou, 121000; \\ ** State Key Laboratory of Explosion Science and Technology, Beijing Institute of Technology, Bei- \\ jing, 100081, China \\ E-mail: pengyajing@126.com
}

\begin{abstract}
The incorporation of $\mathrm{Al}$ particles into explosive formulations has been a subject of ongoing interest because the Al particles can increase the energy content of explosives and tailor the energy release rate. The initial thermal response of micro/nano- spherical Al particles embedded in energetic materials excited by single-pulse laser is investigated by several models, which is crucial for understanding and controlling the thermal ignition mechanism of energetic materials. The thermal relaxation dynamics of electron and lattice in Al particle excited by short pulse (ps) laser are described by the two-temperature model. The characteristics of thermal diffusion of micro/nano-Al particle to the particle environment are analyzed using Fourier law for the long pulse (ns) laser. The influences of laser energy and pulse duration on the thermal response are also examined. The results show that, the thermal diffusion is very important for nano-Al particle during the pulse passage. It can cause the rise of temperature of surrounding energetic materials and thus form the "hot spot" with high temperature and large scale. That can shorten the time of ignition and enlarge the region of ignition. The laser energy and pulse duration are determined to effectively regulate the temperature and size of "hot spot" caused by the nano-Al particles, and the concrete relationships among them are obtained. These results are expected to be a useful indication for adjusting laser ignition (initiation) properties of aluminized explosives by selecting suitable parameters such as particle size, laser pulse duration or laser energy in experiment and practical application.
\end{abstract}

DOI: $10.2961 / \mathrm{jlmn} .2015 .01 .0016$

Keywords: Thermal dynamic response, thermal diffusion, micro/nano-particle, pulsed laser

\section{Introduction}

Aluminized explosives, incorporating the micro- or nano-scale $\mathrm{Al}$ particles into the substrate matrices, are applied widely as underwater ordnance and air weapons due to their numerous outstanding properties such as high energy release rate and chemical reaction rate ${ }^{[1-4]}$. In order to develop more safe and efficient energetic materials, it is essential to understand the formation mechanisms of "hot spot" caused by the embedded micro/nano-Al particle ${ }^{[5,6]}$. Especially, to understand thermal reaction dynamics of energetic materials for laser ignition (initiation), the initial thermal dynamic response (i.e. the formation of "hot spot") of micro/nano-Al particle to pulse laser is the base.

At present, the more researches focus on the performances of chemical reaction and energy release of micro/nano-Al particle in aluminized explosives to reveal the role of additive and the advantage of energetic composites ${ }^{[7-10]}$. However, the initial thermal dynamic response has not been clarified for the micro/nano-Al particle. The influences of Al particles on the thermal decomposition of organic explosives have still not been thoroughly understood [1]. Recently, Yang et al find experimentally that Al nanoparticle causes chemical decomposition of the surrounding oxidizer under pulsed laser heating ${ }^{[1]}$. That means "hot spot" is formed due to laser energy absorption by the $\mathrm{Al}$ particle. Then the initial thermal response properties of $\mathrm{Al}$ particle or the temporal-spatial distribution of temperature caused by it will be an important factor before determining thermal reaction mechanisms.

In this study, different models are applied to investigate the thermal dynamic response of micro/nano-Al particles to ps and ns single pulse laser for analyzing the effect of laser ignition. Thermal diffusion at the interface between particle and surrounding medium, and Gaussian temporal-spatial distribution of laser intensity are taken into account in the models according to the laser pulse duration and the size of system. The generation of hot electron gas, and the electron-phonon coupling are described by the two-temperature model. The thermal transfer into the particle environment and heat propagation in the host medium are modeled by the Fourier law. The thermal relaxation dynamics of the electron and lattice in Al particle, as well as the temporal-spatial temperature of the composite medium, are determined. The effects of laser energy and pulse duration on the thermal response are also discussed. The concrete relationships of temperature and size of "hot spot" with the laser energy and pulse duration are obtained.

\section{Computational model}

Pulsed laser heating the metallic particle involves usually three stages. Free electrons absorb firstly laser energy to attain rapidly high temperatures due to the small heat capacity, and then transfer thermal energy to the material 
lattice due to the electron-phonon scattering. Finally, the thermal energy is transferred into the host medium ${ }^{[12,13]}$.

In order to clarify the mechanisms of thermal response of additives in energetic materials to the pulsed laser, the energetic system with low particle concentrations is usually applied. Previous time-resolved spectroscopy experiments also show the response of nanoparticle to the laser heating is not affected by the other nearby particles when the mass fraction of $\mathrm{Al}$ particle is about $3 \%^{[11,14]}$. Therefore, the thermal dynamic calculations take the model of an isolated spherical particle embedded in a nitrocellulose (NC) sphere as shown in Fig. 1. Nd:YLF pulsed laser $(\lambda=1.053 \mu \mathrm{m})$ with a beam radius of $53 \mu \mathrm{m}$ is chosen according to the previous experiment $^{[11]}$ to heat the system. The laser energy is absorbed only by Al particle because the selective wavelength is almost transparent to NC. As we known, the concrete physical model of laser-particle interactions is different for each case, depending on the laser pulse duration and the characteristic time and size of particle ${ }^{[15,16]}$.

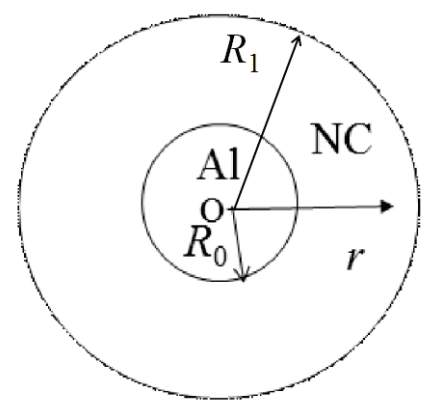

Fig. 1 Geometric model used in calculations

\subsection{Short-pulse excitation of nano-Al particle}

The radius of nano-Al particle $R_{0}=30 \mathrm{~nm}$, and the radius of NC sphere $R_{1}=250 \mathrm{~nm}$. Short-pulse widths are $10 \mathrm{ps}$ and $20 \mathrm{ps}$, respectively. To analyze the thermal response of $\mathrm{Al}$ nanoparticle, the most important thing is whether the thermal diffusion and chemical reaction occur in the environmental medium. Pustovalov ${ }^{[17]}$ shows that the heat exchange time, the characteristic time for heat exchange between a single spherical particle and surrounding medium and the formation of quasi-stationary distribution of temperature in the medium, can be evaluated as $t_{\mathrm{T}}=R_{0}{ }^{2} / 4 D_{\mathrm{m}}$, here $R_{0}, D_{\mathrm{m}}$ is the radius of spherical particle and thermal diffusivity of surrounding medium, respectively. Therefore, the heat transfer from $\mathrm{Al}$ nanoparticle to surrounding $\mathrm{NC}$ can be ignored for the ps regime during the laser heating.

The relaxation time of electron for $\mathrm{Al}$ at room temperature is $0.008 \mathrm{ps}{ }^{[18]}$, which is much shorter than the pulse duration. The hyperbolic transport effect on the electron temperature is unimportant ${ }^{[12]}$. Then the instantaneous internal energy redistribution within the electron gas, and the electron energy transferred to the particle lattice can be described by the two-temperature model ${ }^{[19,20]}$. The heat transfer is described by one dimensional equation due to the spherical symmetry.

$$
\begin{gathered}
C_{e}\left(T_{e}\right) \frac{\partial T_{\mathrm{e}}(t)}{\partial t}=-G\left(T_{e}-T_{l}\right)+p(t) \\
C_{l} \frac{\partial T_{l}(t)}{\partial t}=G\left(T_{e}-T_{l}\right) \\
C_{e}\left(T_{e}\right)=\gamma T_{e} \\
p(t)=a e^{-b\left(t-t_{0}\right)^{2}}
\end{gathered}
$$

where $T_{\mathrm{e}}, T_{1}$ is respectively the temperature of electron and lattice, $G$ is the electron-phonon coupling constant, $C_{\mathrm{e}}\left(T_{\mathrm{e}}\right)$ is the temperature-dependent electron heat capacity, $\gamma$ is a constant, $C_{1}$ is the lattice heat capacity, $p(t)$ denotes the instantaneous power density absorbed by particle, here $a$ and $b$ are the parameters dependent on the laser and system regime ${ }^{[21,22]}$, and $t_{0}$ is a time parameter used to determine the moment approaching the peak absorption intensity. The temperature of electron is assumed to be constant through the nanoparticle because the heat transfer between electron and electron is very fast (femtosecond order). The initial conditions are as follows:

$$
\left.T_{e}\right|_{t=0}=\left.T_{l}\right|_{t=0}=T_{0}
$$

\subsection{Long-pulse excitation of nano-Al particle}

The long-pulse duration takes 10ns. For the nanosecond single-pulse laser, the heat exchange between electron and lattice is completed during the pulse passage, that is, the temperatures of electron and lattice are equal at any time ${ }^{[15]}$. Here, the pulse duration is larger than the heat exchange time $(\sim 1 \mathrm{~ns})$ of particle-medium. The heat transfer from $\mathrm{Al}$ nanoparticle to surrounding $\mathrm{NC}$ cannot be ignored. So the Fourier law is used to describe the temperature distribution of Al nanoparticle and surrounding NC.

$$
\begin{gathered}
\rho_{A l} c_{A l} \frac{\partial T_{A l}(r, t)}{\partial t}=k_{A l} \frac{\partial^{2} T_{A l}(r, t)}{\partial r^{2}}+\left.\frac{S}{V} k_{m} \frac{\partial T_{m}(r, t)}{\partial r}\right|_{r=R_{0}}+p(t) \\
\frac{\partial T_{m}(r, t)}{\partial t}=D_{m} \frac{\partial^{2} T_{m}(r, t)}{\partial r^{2}}
\end{gathered}
$$

Here, $\rho_{\mathrm{Al}}, c_{\mathrm{Al}}$ is the density and specific heat capacity of $\mathrm{Al}$; $T_{\mathrm{m}}, K_{\mathrm{m}}$ is the temperature and thermal conductivity of $\mathrm{NC}$, respectively. The second item on the right side in eq. (6) denotes transferred heat from Al sphere surface into surrounding NC. The $S, V$ is the surface area and volume of $\mathrm{Al}$ sphere, respectively. Eq. (7) describes the temporal-spatial distribution of temperature in NC. An important characteristic of laser heating solid metallic nanoparticle is that the heated particle may reach directly the vaporization temperature without melting ${ }^{[17]}$. So the latent heat of fusion of $\mathrm{Al}$ is not considered. The chemical reaction of materials is excluded in order to clarify the initial thermal response properties. The initial and boundary conditions are

$$
\begin{gathered}
T_{\mathrm{Al}}(r, 0)=T_{\mathrm{m}}(r, 0)=T_{0} \\
\left.\frac{\partial T_{\mathrm{Al}}(r, t)}{\partial r}\right|_{r=0}=0 \\
\left.k_{A l} \frac{\partial T_{\mathrm{Al}}(r, t)}{\partial r}\right|_{r=R_{0}}=\left.k_{m} \frac{\partial T_{\mathrm{m}}(r, t)}{\partial r}\right|_{r=R_{0}} \\
T_{\mathrm{Al}}\left(R_{0}, t\right)=T_{\mathrm{m}}\left(R_{0}, t\right) \\
\left.T_{\mathrm{m}}(r, t)\right|_{r=R_{1}}=T_{0}
\end{gathered}
$$

\subsection{Micro-Al particle excited by pulsed laser}


When the size of $\mathrm{Al}$ particle is of micrometer order and it is comparable to the laser beam radius, the spatial distribution of laser intensity cannot be ignored. The physical equations are

$$
\begin{gathered}
\rho_{A l} c_{A l} \frac{\partial T(r, t)}{\partial t}=k_{A l} \frac{\partial^{2} T(r, t)}{\partial r^{2}}+\left.\frac{S}{V} k_{m} \frac{\partial T_{m}(r, t)}{\partial r}\right|_{r=R_{0}}+p(r, t) \\
\frac{\partial T_{m}(r, t)}{\partial t}=D_{m} \frac{\partial^{2} T_{m}(r, t)}{\partial r^{2}}
\end{gathered}
$$

The absorbed instantaneous power density including of Gaussian spatial distribution is

$$
p(r, t)=a e^{-b\left(t-t_{0}\right)^{2}} e^{\left(-2 r^{2} / w^{2}\right)}
$$

Eq. (9) shows both the thermal diffusion and optical absorption are taken into account in the heat transfer. The initial and boundary conditions are the same as those listed in the section 2.2. The finite difference method is used to solve the differential equations.

\section{Results and discussions}

The pulse energy in the above three models is chosen respectively as $1 \mu \mathrm{J}, 50 \mu \mathrm{J}$, and $10 \mathrm{~J}$ in order to obtain the adequate temperature. The parameters used in the calculations are listed in Table 1 and Table 2. The initial temperature $T_{0}=300 \mathrm{~K}$, and the time parameter $t_{0}=4 / 3 t_{\mathrm{p}}{ }^{[13,21]}, t_{\mathrm{p}}$ is the pulse duration of laser. The computing time goes $0-3 t_{0}$ for the ps regime and $0-2 t_{0}$ for the ns regimes.

\begin{tabular}{|c|c|c|c|c|c|c|c|c|}
\hline $\begin{array}{l}\rho_{\mathrm{Al}} \\
(\mathrm{kg} / \\
\left.\mathrm{m}^{3}\right)\end{array}$ & $\begin{array}{l}c_{\mathrm{Al}} \\
(\mathrm{J} / \\
\mathrm{kg} . \\
\mathrm{K}) \\
\end{array}$ & $\begin{array}{l}k_{\mathrm{Al}} \\
(\mathrm{w} / \\
\mathrm{m} \cdot \\
\mathrm{K}) \\
\end{array}$ & $\begin{array}{l}\quad c_{1} \\
(\mathrm{~J} / \mathrm{kg} \\
\mathrm{K})\end{array}$ & $\begin{array}{c}\gamma^{[23]} \\
\left(\mathrm{J} / \mathrm{m}^{3} \cdot \mathrm{K}\right. \\
\left.{ }^{3}\right)\end{array}$ & $\begin{array}{l}c_{\mathrm{e}}^{[24]} \\
(\mathrm{J} / \mathrm{kg} . \\
\mathrm{K})\end{array}$ & $\begin{array}{l}G^{[25]} \\
(\mathrm{w} / \mathrm{m} \\
\left.{ }^{3} \cdot \mathrm{K}\right)\end{array}$ & $\begin{array}{l}k_{\mathrm{m}} \\
(\mathrm{w} / \mathrm{m} \\
\cdot \mathrm{K})\end{array}$ & $\begin{array}{l}D_{\mathrm{m}} \\
\left(\mathrm{m}^{2} / \mathrm{s}\right. \\
)\end{array}$ \\
\hline 2700 & $\begin{array}{l}90 \\
3\end{array}$ & $\begin{array}{l}23 \\
7\end{array}$ & $\begin{array}{l}2.4 \times 1 \\
0^{6}\end{array}$ & 91.2 & $\begin{array}{l}4.1 \times 1 \\
0^{4}\end{array}$ & $\begin{array}{l}23 \times 1 \\
0^{16}\end{array}$ & 0.229 & $\begin{array}{l}1.78 \\
\times 10^{-7}\end{array}$ \\
\hline
\end{tabular}

Table 1 Values of parameters of $\mathrm{Al}$ and $\mathrm{NC}$ used in the calculations ${ }^{[22]}$

Table 2 Parameters of instantaneous power density absorbed by Al particle. The $a$ and $b$ are the coefficients of $p(\mathrm{t})$, which are dependent on the laser and system; $t_{0}$ is the moment of approaching peak power density.

\begin{tabular}{cccc}
\hline $\begin{array}{c}\text { Pulse } \\
\text { duration }\end{array}$ & $\begin{array}{c}a \\
\left(\mathrm{w} / \mathrm{m}^{3}\right)\end{array}$ & $\begin{array}{c}b \\
\left(1 / \mathrm{s}^{2}\right)\end{array}$ & $\begin{array}{c}t_{0} \\
(\mathrm{~s})\end{array}$ \\
\hline $10 \mathrm{ps}$ & $2.882 \times 10^{18}$ & $2.773 \times 10^{22}$ & $1.333 \times 10^{-11}$ \\
$20 \mathrm{ps}$ & $1.441 \times 10^{18}$ & $6.931 \times 10^{21}$ & $2.667 \times 10^{-11}$ \\
$10 \mathrm{~ns}$ & $2.157 \times 10^{15}$ & $2.773 \times 10^{16}$ & $1.333 \times 10^{-8}$ \\
\hline
\end{tabular}

\subsection{Thermal relaxation effect of nano-Al particle to short-pulse laser}

Figure 2 shows the time profiles of electron temperature and instantaneous power density absorbed by Al nanoparticle excited by $10 \mathrm{ps}$ pulsed laser. The dotted line denotes the power density. The solid line represents the electron temperature in Al nanoparticle. From the Fig. 2, the tem- poral evolution of the electron temperature can be obviously divided into two time periods: the electron heating time and the electron cooling time. When the pulse laser irradiates onto the composite, the electrons absorb laser energy and theirs temperature rise gradually. The maximum temperature of electrons is reached with a delay of $10 \mathrm{ps}$ after the peak power density. The electron heating time lasted about 20ps. Then, the electrons are cooled due to transferring heat to the lattice, resulting in a decay of the electron temperature. The electron cooling time is about 10ps. after that, the electron temperature reaches thermal equilibrium with the lattice temperature. Here, the temperature response of

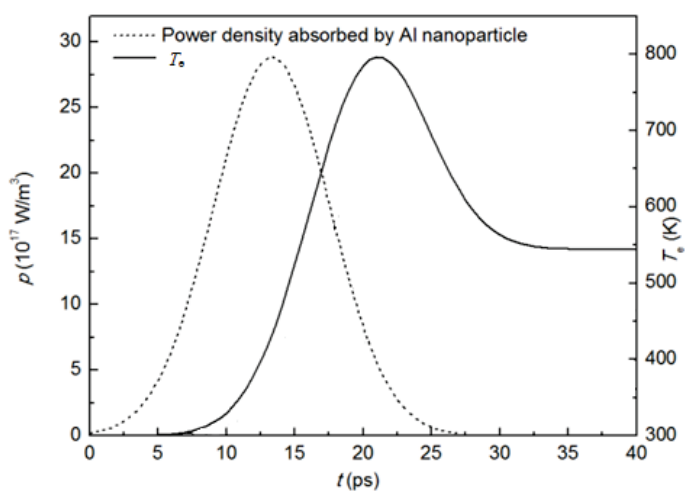

Fig. 2 Temperature dynamics of electron (solid) in Al nanoparticle (30nm radius) and laser power density (dashed) absorbed by Al nanoparticle vs time under the excitation of 10 ps pulsed laser with energy $E=1 \mu \mathrm{J}$.

electrons is not very sharp due to the high electron-lattice coupling constant ${ }^{[26]}$. The evolution of lattice temperature is displayed in Fig. 3. Comparing with the electron temperature, the lattice temperature always rises via coupling heat from electrons. The temperature of electrons exceeds that of lattice before equilibrium. The lattice thermalization time, the time for electrons and lattice to reach thermal equilibrium, is about 30ps. The thermal response experiences of electrons and lattices are identified as the Al target ablated by fs laser ${ }^{[15,27]}$.

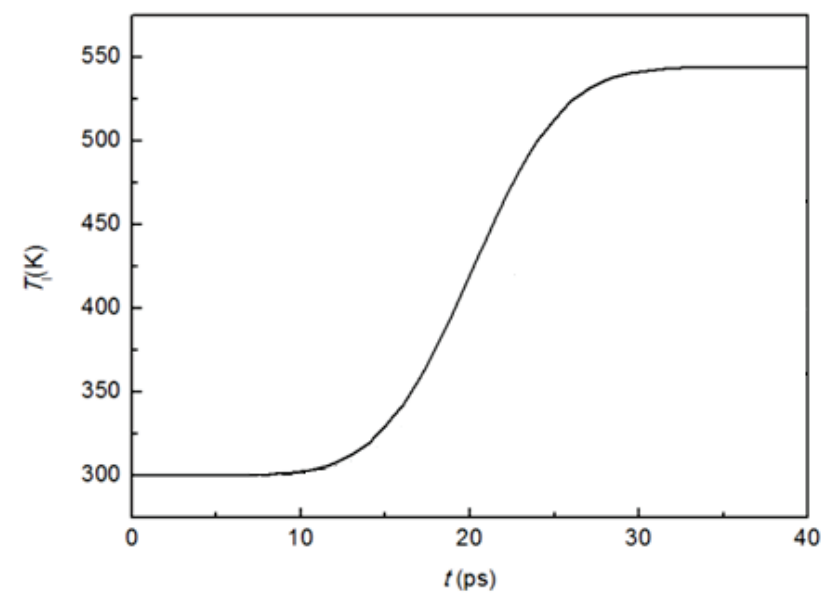

Fig. 3 Lattice temperature dynamics of $\mathrm{Al}$ nanoparticle (30nm radius) excited by $10 \mathrm{ps}$ pulsed laser with energy $E=1 \mu \mathrm{J}$. 
Figure 4 presents the evolutions of the electron and lattice temperatures in a nano-Al particle $\left(R_{0}=30 \mathrm{~nm}\right)$ excited by 20 ps pulsed laser. When the pulse duration becomes long, the peak power density absorbed by the Al nanoparticle decreases according to the parameter $a$ in Table 2. So the maximum temperature of electrons and the equilibrium temperature of electrons and lattice become low compared to that for $10 \mathrm{ps}$ regime. Also, the electron heating time (about 40ps) and electron cooling time (about 20ps) become long. The equilibrium of temperature between electrons and lattice takes up to about $60 \mathrm{ps}$. So the long pulse duration increases the lattice thermalization time and slows down the process of thermal response.

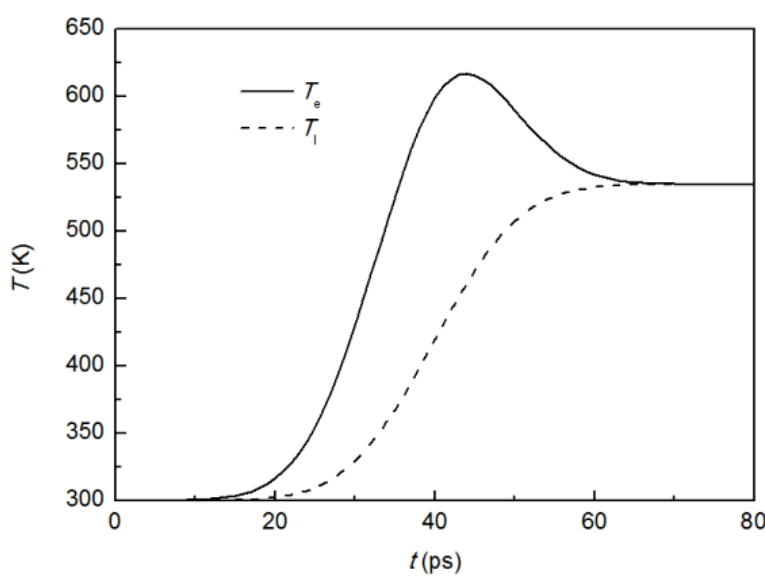

Fig. 4 The dynamics of electron and lattice temperatures in $\mathrm{Al}$ nanoparticle of radius $R_{0}=30 \mathrm{~nm}$ heated by $20 \mathrm{ps}$ pulsed laser with energy $E=1 \mu \mathrm{J}$.

Figure 5 indicates the influences of laser energy on the thermal dynamic properties of electrons and lattice. As the increase of laser energy, the peak power density absorbed by nanoparticle also is increased. So the temperatures of electrons and lattice increase, the electron heating time and electron cooling time slightly become long, and the lattice thermalization time correspondingly increases. This is accordant with that the higher laser power gives slower decay of electron temperature ${ }^{[28-30]}$.

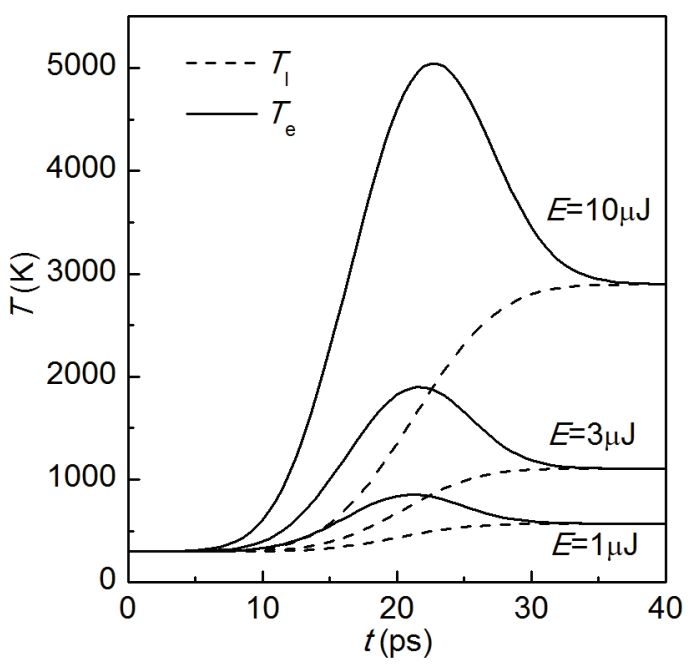

Fig. 5 Thermal dynamic response of electrons and lattice in $\mathrm{Al}$ nanoparticle of radius $R_{0}=30 \mathrm{~nm}$ excited by $10 \mathrm{ps}$ pulsed laser with different energy.

\subsection{Thermal response of Al nanoparticle under long- pulse laser excitation}

The time evolution of temperature in $\mathrm{Al}$ nanoparticle and surrounding NC predicted by Fourier law is shown in Fig. 6 for the 10ns pulse irradiation. The dash-dotted line in that figure denotes the instantaneous power density absorbed by Al nanoparticle. The solid curve shows the temperature of $\mathrm{Al}$ nanoparticle arises descending after reaching the maximum due to transferring heat to the surrounding

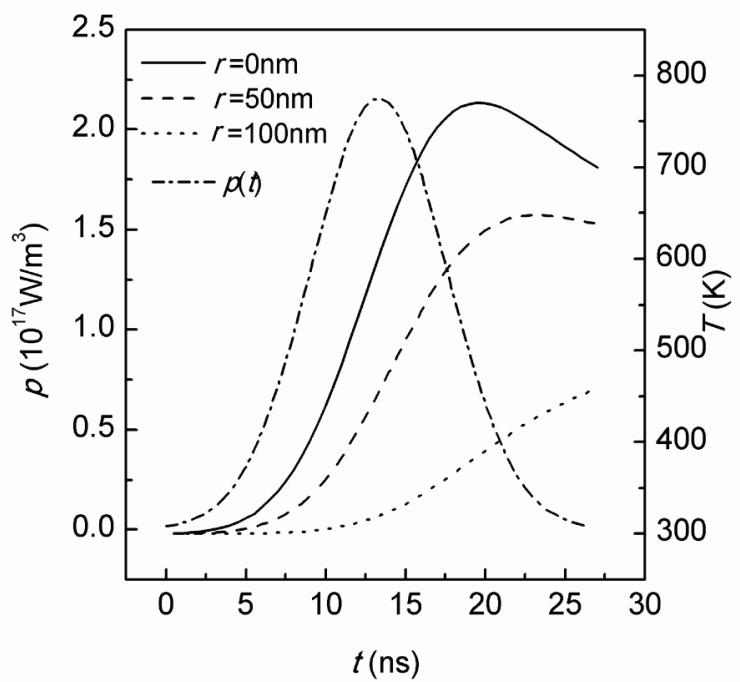

Fig. 6 The instantaneous temperature at different spatial positions and the laser power density absorbed by $\mathrm{Al}$ nanoparticle when excited by $10 \mathrm{~ns}$ pulse laser with energy $E=50 \mu \mathrm{J}$.

NC. The temperature at the position near the heating center in $\mathrm{NC}$ (dashed line) exhibits the same change trend with the temperature of Al nanoparticle, while that at the farther spot (dotted line) gradually increase before the end of pulse. Fig. 7 shows the spatial temperature distribution of composite medium at different time. The time values are selected

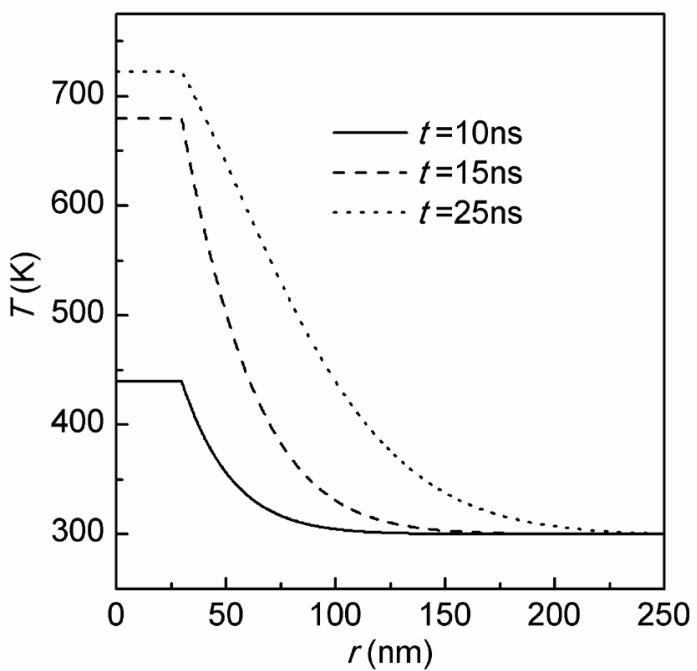

Fig. 7 The spatial temperature distribution of $\mathrm{Al}$ nanoparticle and $\mathrm{NC}$ under the excitation of $10 \mathrm{~ns}$ pulsed laser with $E=50 \mu \mathrm{J}$ at the end of pulse $\left(t=2 t_{0}\right)$. 
as that before, near, and after the peak power density respectively so as to display comprehensively the characteristics of temperature distribution during the pulse duration. As can be seen, before reaching the maximum power density(10-15ns), the temperature of Al nanoparticle increases rapidly due to the increase of absorbed laser energy. While after the peak power density (15-25ns), the increase in temperature reduces because that the absorbed power density decreases gradually during that time. The main feature for ns pulse heating is the heat diffusion from the nanoparticle surface to the surrounding $\mathrm{NC}$ medium. The temperature through the $\mathrm{Al}$ nanoparticle (flat part) is almost equal because of its large thermal conductivity, while the declining part represents the temperature of $\mathrm{NC}$ due to transferring heat from $\mathrm{Al}$ particle. The length of thermal diffusion $(200 \mathrm{~nm})$ is larger than that of the nanoparticle $(30 \mathrm{~nm}$ radius). This program has also been applied as a part to calculate the chemical reaction diameters caused by Al nanoparticle excited by ns pulse laser in other literature ${ }^{[21]}$, and the validity has been proven by the relevant experiment data.

Fig. 8 presents the 3-D temperature features in the nano-composite medium as the functions of laser energy and the distance from nanoparticle center to analyze the influences of pulse energy on the thermal response. It can be seen that the increase of laser energy will increase the temperature of the composite medium and expand the heated region of surrounding NC. In order to obtain further the relationship among them, the spatial temperature caused by different laser energy is shown in Fig. 9. Here, the temperature of "hot spot" $T_{\mathrm{h}}$ is represented by the maximum temperature. The size of "hot spot" $r_{\mathrm{h}}$ is defined as the radius of area with the temperature beyond the ignition temperature $T_{\mathrm{p}}$, which is because that only the "hot spot" triggering chemical reaction can possibly realize ignition.

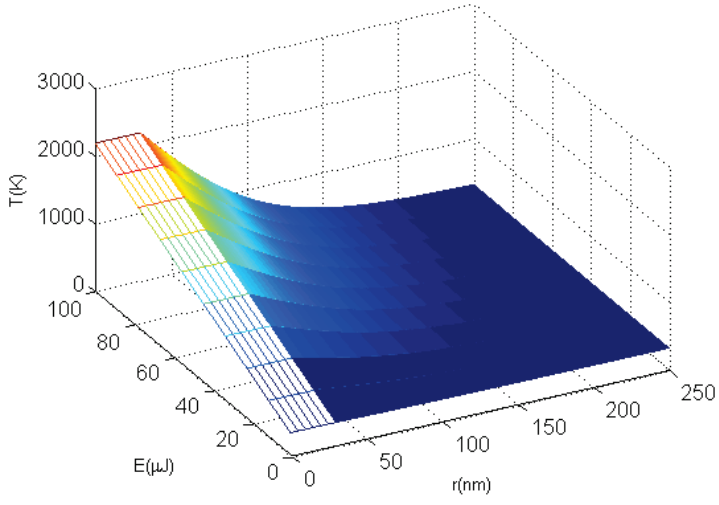

Fig. 8 The 3-D temperature distribution of medium as the functions of laser energy and the distance from nanoparticle center at the end of 10ps pulse $\left(t=2 t_{0}\right)$.

Then for the NC with constant ignition temperature $\left(T_{\mathrm{p}}=440 \mathrm{~K}\right)$, the temperature and size of the "hot spot" caused by $\mathrm{Al}$ nanoparticle increase with the increasing laser energy. That is, more NC can reach the ignition threshold and more energy can be released at the same time. Accordingly, the increase of laser energy will enhance the ignition velocity and reduce the ignition time. Figs.10(a) and 10(b) show the influences of laser energy on the temperature and size of "hot spot" caused by Al nanoparticle with radius of

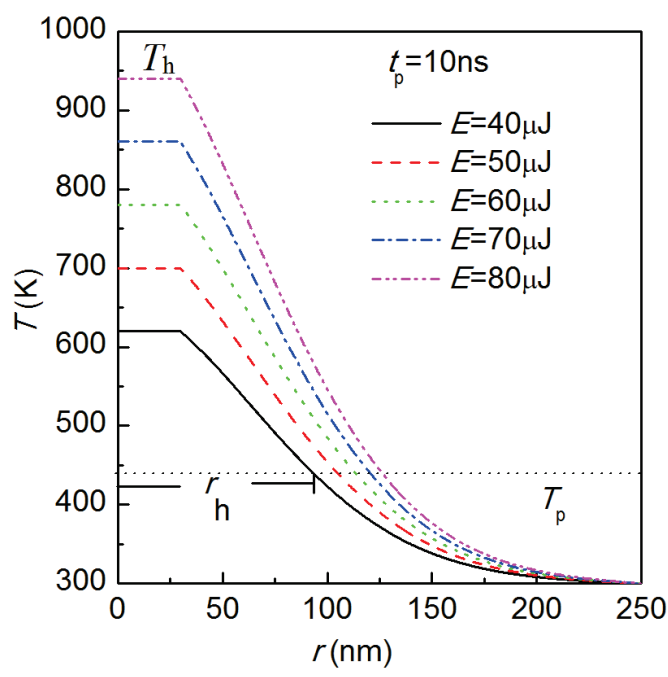

Fig. 9 Spatial temperature distribution of composite medium excited by different laser energy at $t=2 t_{0}$. The $T_{\mathrm{h}}, r_{\mathrm{h}}$ represents respectively the temperature and size of "hot spot", and $T_{\mathrm{p}}$ is the ignition temperature of $\mathrm{NC}$.
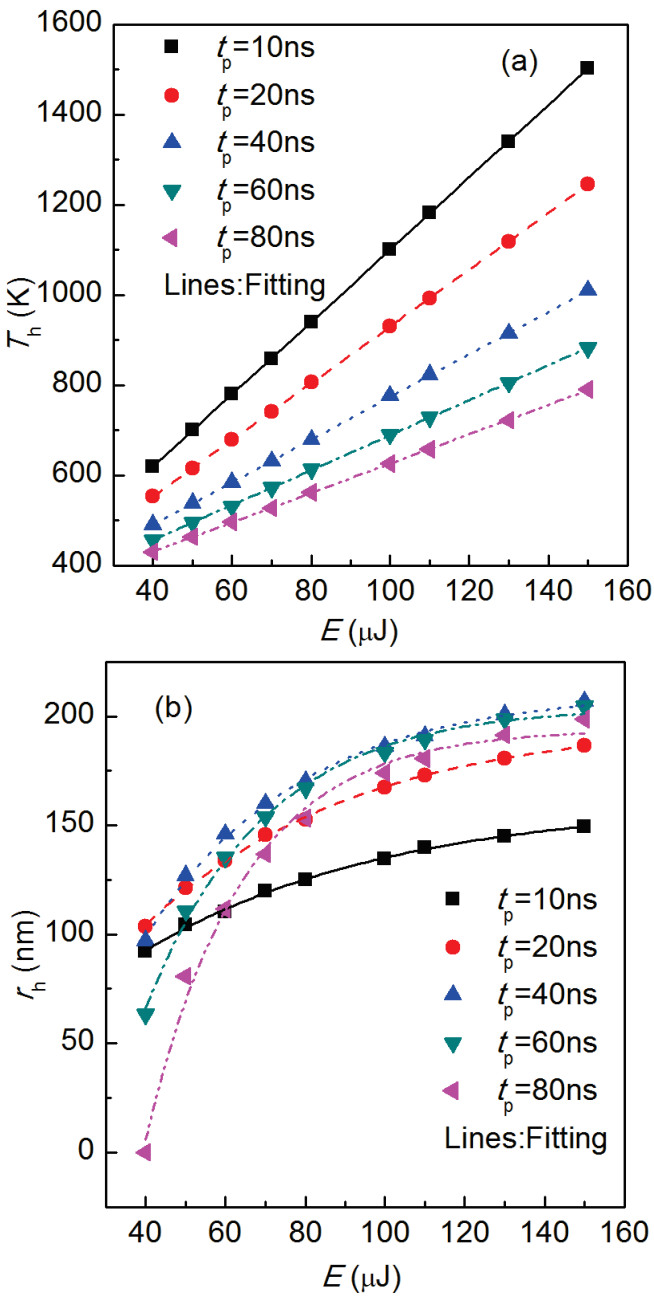

Fig. 10 Influences of laser energy on the temperature (a) and size (b) of "hot spot" caused by Al nanoparticle with radius of $30 \mathrm{~nm}$. The symbols are the calculated data from Fig. 9, and the lines are the fitting to those data. 
$30 \mathrm{~nm}$, respectively. The symbols represent the calculated data, and the lines represent the fitting to those data. The fitting equation to the $E-T_{\mathrm{h}}$ is expressed as

$$
T_{h}=a_{1}\left(t_{p}\right)+b_{1}\left(t_{p}\right) E,
$$

and the pulse duration-dependent parameters are given by fitting the obtained parameters as

$$
\begin{aligned}
& a_{1}\left(t_{p}\right)=296.3+0.219 t_{p} \\
& b_{1}\left(t_{p}\right)=7.94-0.06 t_{p}
\end{aligned}
$$

The fitting equation to the $E-r_{\mathrm{h}}$ is shown as followed

$$
r_{h}=a_{2}\left(t_{p}\right)+b_{2}\left(t_{p}\right) e^{\left(-E / c_{2}\left(t_{p}\right)\right)},
$$

With the pulse duration-dependent parameters are given by fitting as

$$
\begin{aligned}
& a_{2}\left(t_{p}\right)=142.8+2.78 t_{p} \\
& b_{2}\left(t_{p}\right)=-171.8+3.45 t_{p} . \\
& c_{2}\left(t_{p}\right)=63.8-0.53 t_{p}
\end{aligned}
$$

As can be seen, a linear increase relationship exists between the temperature of "hot spot" and the increasing laser energy, and the slope linearly decreases as the increase of pulse duration (see (13)). The size of "hot spot" satisfies exponential growth for the increasing energy. The coefficients are all pulse duration-dependent.

Fig. 11 presents the 3-D temperature features in composite medium in the effects of pulse duration and the distance from nanoparticle center. It shows the short-pulse duration will increase the maximum temperature of medium. That is because the decrease of pulse duration increases the peak power density absorbed by nanoparticle according to the parameter $a$ in $p(\mathrm{t})$ as shown in Table 2, and consequently raises the equilibrium temperature at the end of the pulse. We know that the released heat from chemical reaction increases with the increase of temperature according to the Arrhenius law (Q e (-Ea/RT)). So the formation of "hot spot" with high temperature can easily lead to ignite due to releasing more energy.

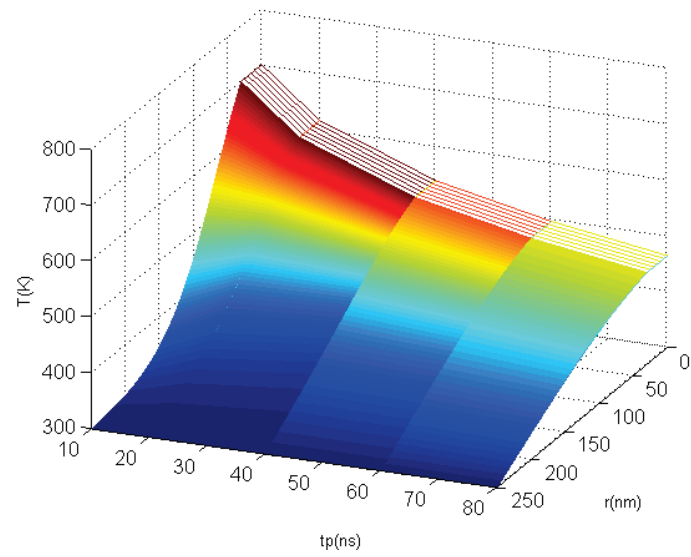

Fig. 11 The 3-D temperature distribution of medium as the functions of pulse duration and the distance from nanoparticle center at the end of pulse $\left(t=2 t_{0}\right)$ with energy of $50 \mu \mathrm{J}$.

To explore the effects of pulse duration on the temperature and size of "hot spot", the spatial temperature distribu

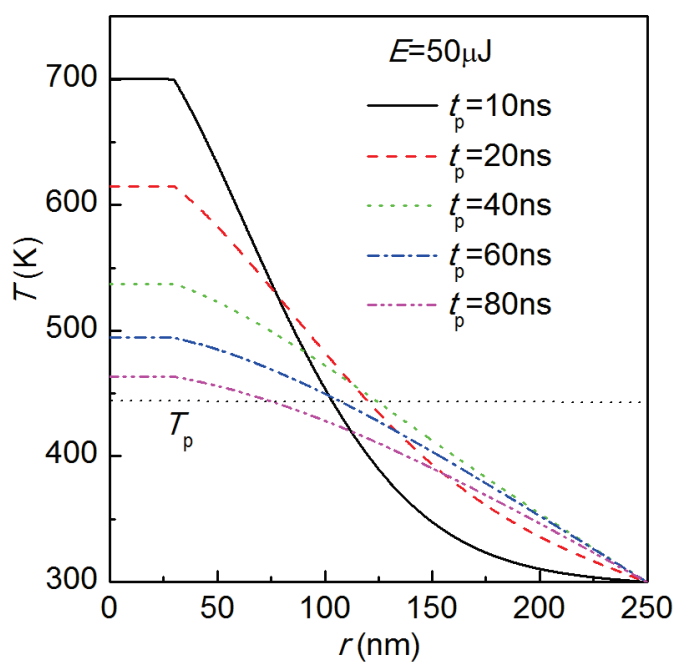

Fig. 12 Spatial temperature distribution at of composite medium including Al nanoparticles (radius of $30 \mathrm{~nm}$ ) affected by laser pulse duration at $t=2 t_{0}$.
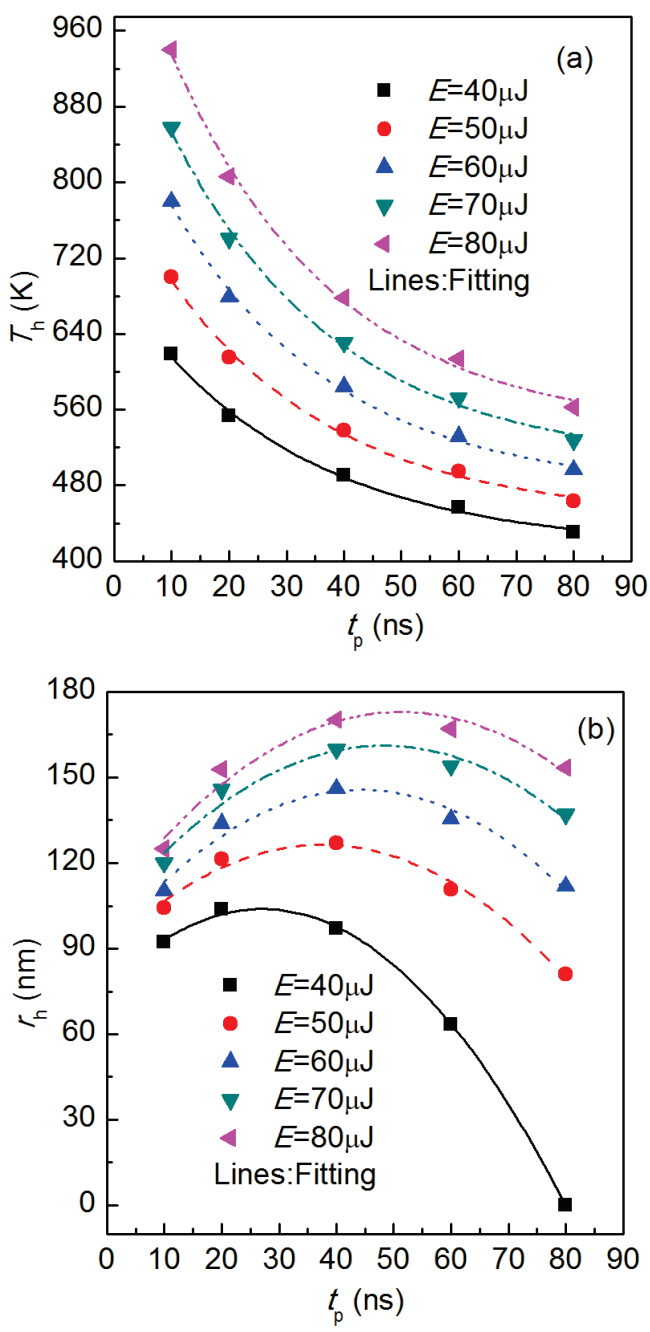

Fig. 13 Temperature (a) and size (b) of "hot spot" against the pulse duration. The symbols represent the calculated data from Fig. 12. The Lines are the fitting to those data. 
tion caused by $30 \mathrm{~nm}$ Al nanoparticle excited by different pulse duration is shown in Fig. 12. Obviously, the temperature of "hot spot" decreases gradually and the size of "hot spot" increases first and then decreases against the pulse duration. The specific change relationship can be obtained by fitting the calculated data (symbols) as shown in Figs. 13(a) and 13(b). The fitting equation to the $T_{\mathrm{h}}-t_{\mathrm{p}}$ is written as

$$
T_{h}=a_{3}(E) e^{-t_{p} / b_{3}(E)}+c_{3}(E),
$$

Where, the laser energy-dependent parameters are fitted as:

$$
\begin{aligned}
& a_{3}(E)=-3.73+7.16 E \\
& b_{3}(E)=36.6-0.24 E \\
& c_{3}(E)=294.7+3.00 E
\end{aligned} .
$$

The fitting equation to the $r_{\mathrm{h}}-t_{\mathrm{p}}$ is written as

$$
r_{h}=a_{4}(E)+b_{4}(E) t_{p}+c_{4}(E) t_{p}^{2},
$$

Where, the energy-dependent parameters satisfy the following fitting equations:

$$
\begin{aligned}
& a_{4}(E)=53.35+0.68 E \\
& b_{4}(E)=1.22+0.02 E \\
& c_{4}(E)=-0.04+2.32 \times 10^{-4} E
\end{aligned} .
$$

The fitting results show that the decay of temperature of "hot spot" with the increase of pulse duration can be approximately fitted by an exponential function, while a quadratic-polynomial fit provides a good approximation to the relationship between the size of "hot spot" and pulse duration. The $r_{\mathrm{h}}$ thus reaches a maximum value when the pulse duration and laser energy approximately meet the following equation

$$
t_{p}=\frac{1}{4}(61+E) .
$$

That means, for the high-energy laser, the long pulse duration should be chosen to get the maximum size of "hot spot". However, it is worth noting that these fitting formulas are valid only for a range of energy and pulse duration. For example, the parameter $b 1$ in equation (12) should be positive to insure that the slope is positive. The pulse duration $t_{\mathrm{p}}$ thus should be smaller than $\sim 130 \mathrm{~ns}$ according to equation (13).

Figs. 14 (a) and 14(b) show the influences of laser energy together with pulse duration on the temperature and size of "hot spot", respectively. It can be seen that the temperature of "hot spot" will increase monotonically with the increase of laser energy and (or) the decrease of pulse duration. However, the size of "hot spot" will appear a maximum value in the regulation of energy and pulse width. The calculation results predict the concrete relationship among the temperature and size of "hot spot", laser energy, and pulse duration. That is expected to be a useful indication for adjusting the properties of laser igniting nanoenergetic materials by selecting suitable laser energy and pulse duration in practical application.
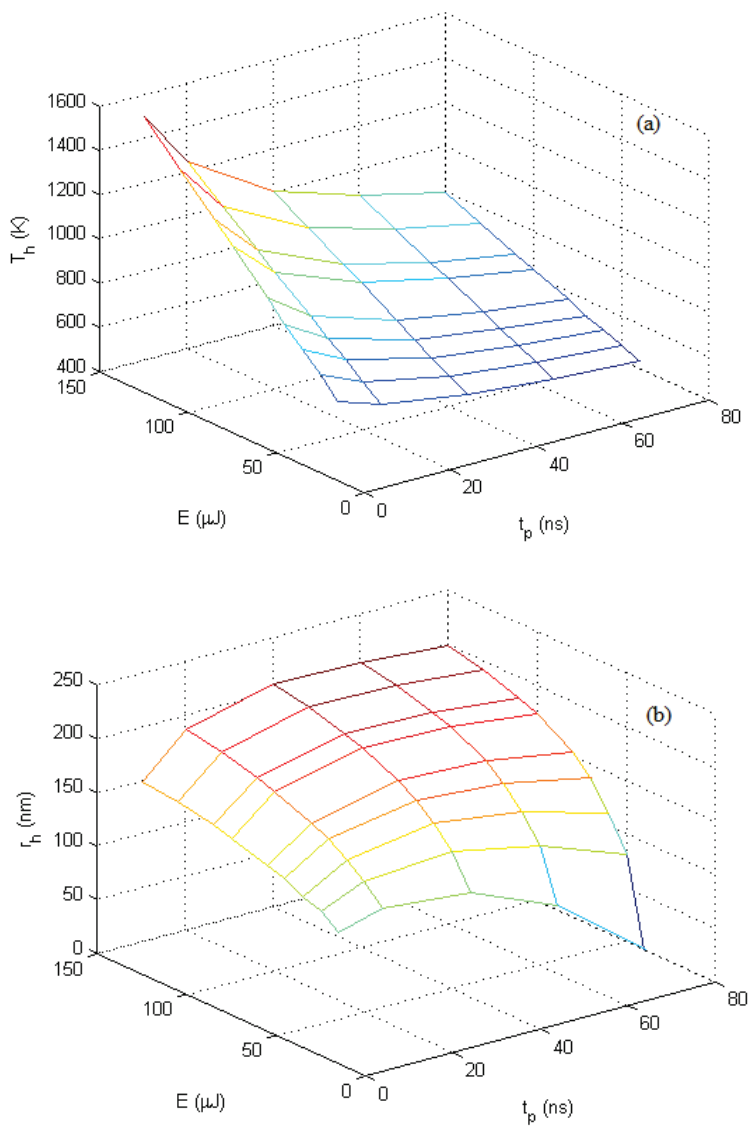

Fig. 14 Variations of temperature (a) and size (b) of "hot spot" affected by laser energy together with pulse duration at the end of the laser pulse $\left(t=2 t_{0}\right)$.

\subsection{Temporal-spatial temperature properties of micro- Al particle under pulsed laser excitation}

The calculation is given for micro $\mathrm{Al}$ particle with radius of $10 \mu \mathrm{m}$. The energy of laser is $10 \mathrm{~J}$. The radius of NC sphere R1 is selected as $70 \mu \mathrm{m}$ to adapt memory of computer. Fig. 15 is the topography of Gaussian temporal-spatial distribution of instantaneous power density absorbed by micro-Al particle excited by $10 \mathrm{~ns}$ pulse. The temporal

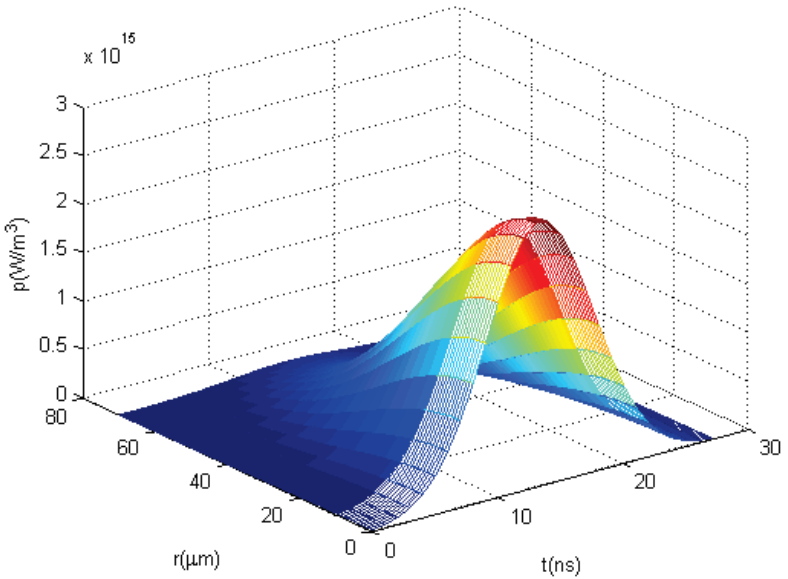

Fig. 15 The Gaussian temporal-spatial distribution of instantaneous power density absorbed by the $\mathrm{Al}$ micro-particle excited by $10 \mathrm{~ns}$ pulsed laser with energy of $10 \mathrm{~J}$. 
temperatures at different positions in the composite medium are shown in Fig. 16. We can see that the temperatures in the interior of $\mathrm{Al}$ particle are not equal (solid and dashed lines) due to incorporating the Gaussian spatial distribution of pulse intensity. Only the temperature of $\mathrm{NC}$ medium very close to the Al particle gradually increases. The spatial variation of temperature in the composite medium is indicated in the Fig. 17. The inset represents an enlargement of the part marked by the circle to observe clearly the scale of thermal diffusion. Obviously, the length of thermal diffusion for the particle with radius of $10 \mu \mathrm{m}$ is about $0.3 \mu \mathrm{m}$. It

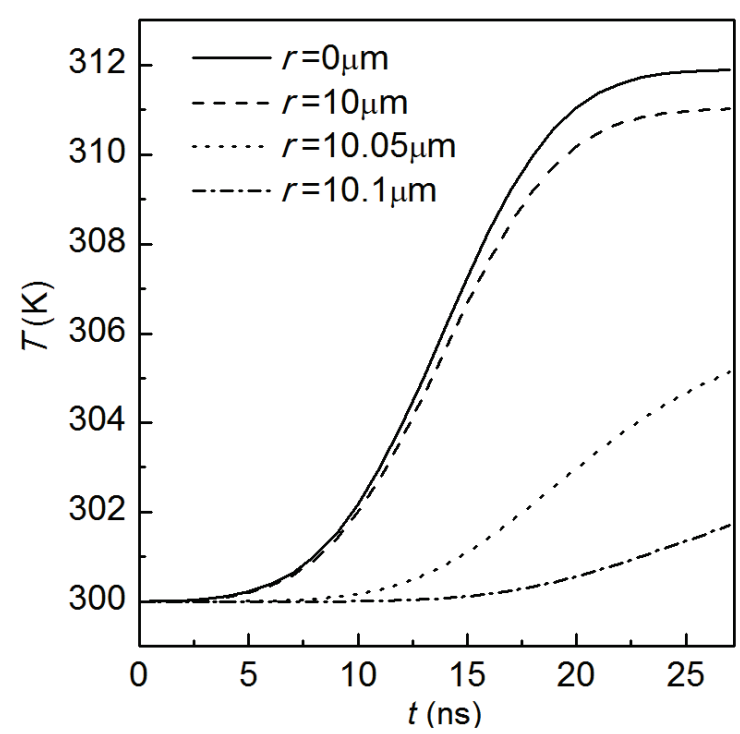

Fig. 16 Temporal temperatures at different spatial positions in the composite medium excited by $10 \mathrm{~ns}$ pulsed laser with energy of $10 \mathrm{~J}$.

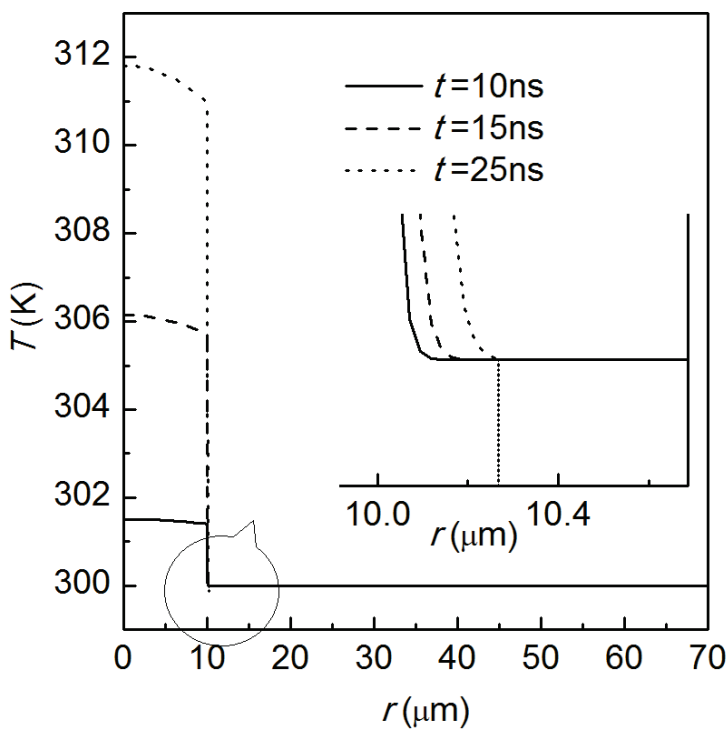

Fig. 17 The spatial temperature distribution of $\mathrm{Al}$ nanoparticle and $\mathrm{NC}$ under the excitation of 10ns pulsed laser with energy of $10 \mathrm{~J}$ at the end of pulse $\left(t=2 t_{0}\right)$. The inset shows an enlarged part marked by circle to observe the length of thermal diffusion.

shows the size of thermal diffusion is very small compared to the size of the micro-Al particle. So the thermal diffu- sion might be ignored during the pulse duration in the thermal analysis of micro-scale particle. This is identified as the previous estimation of thermal diffusion properties between particle and environment ${ }^{[17]}$. That means the thermal response of micro-particle is not sensitive to the pulsed laser compared to that of the nanoparticle. In this case, the temperature of forming "hot spot" is low and its size is small even for the same maximum temperature with nanoparticle. Therefore, the ignition is not prone to occur. The Figs. 18 (a) and 18(b) give the influences of pulse energy and pulse duration on the temperatures of composite medium. It can be seen that altering the pulse energy or
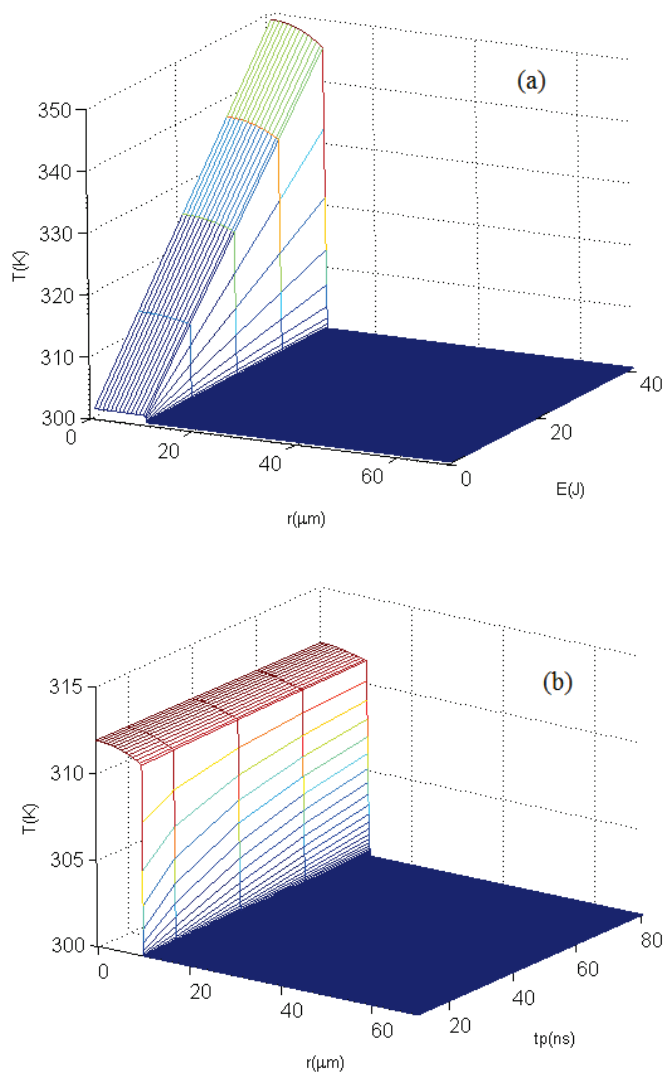

Fig. 18 The temperature distribution of medium heated by $10 \mathrm{~ns}$ pulsed laser with different energy (a) and that heated by 10J pulsed laser with different pulse duration (b) at the end of pulse $\left(t=2 \mathrm{t}_{0}\right)$.

pulse duration will not effectively increase the heat diffusion into the surrounding NC to cause the chemical reaction for the micro-regime. Therefore, reducing Al particle size from micro- to nano-meter will greatly improve the ignition (initiation) sensitivity of energetic materials.

\section{Conclusions}

The heating kinetics of micro/nano-scale Al particle embedded in the energetic materials under the excitation of ps and ns single-pulse laser is investigated by different models. The nonequilibrium relaxation dynamics is analyzed by the two-temperature model for the nano-Al particle excited by ps laser pulse. Reducing pulse duration can shorten effectively the coupling time between electrons and 
lattice, while increasing pulse energy can slow down the coupling process. The Fourier law, including the thermal diffusion at the interface between $\mathrm{Al}$ particle and surrounding $\mathrm{NC}$, and the absorbed instantaneous power density by Al particle, is employed to explore the thermal response to ns pulse. The temporal-spatial distribution of temperature shows that the thermal diffusion into the energetic materials is very important for the nano-particle. The incorporated nano-scale $\mathrm{Al}$ particle can help energetic materials to form the "hot spot" with high temperature and large scale and the nanoenergetic materials is more sensitive to laser ignition or initiation. The pulse duration and laser energy can regulate effectively the temperature and size of "hot spot" formed by nanoparticle. The temperature of "hot spot" could be linearly increased by increasing laser energy, and be exponentially reduced by increasing pulse duration. The size of "hot spot" exhibits exponential growth with increasing laser energy, and increase first and then decrease with the increase of pulse duration. The concrete relations with laser energy and pulse duration for temperature and size of "hot spot" are shown by the 3-D topography. These results are expected to provide significate theory basis for experiment and practical application to promoting or restraining the ignition (initiation) of nano-composite energetic materials by adjusting the pulse duration and energy.

\section{Acknowledgments}

The authors acknowledge the supports of National Science Foundation of China (No.21203012), the fund of the Excellent Talents Support Program of institutions of higher learning of Liaoning province (LJQ2013118), and the opening project of State Key Laboratory of Explosion Science and Technology (Beijing Institute of Technology) (KFJJ14-8M)

\section{References}

[1] C. H. Hou, X. H. Geng, C. W. AN, J. Y. Wang, W. Z. $\mathrm{Xu}$, and X. D. Li: Central European Journal of Energetic Materials, 10, (2013) 123.

[2] Y. J. Peng, Y. H. Wang, Y. Q. Yang, and D. D. Dlott: $J$. Nanopart Res., 12, (2010) 777.

[3] C. Rossi, K. L. Zhang, D. Estève, P. Alphonse, P. Tailhades, and C. Vahlas: Journal of Microelectromechanical Systems, 16, (2007) 919.

[4] R. Singh, N. Badi, M. Hobosyan, and K. Martirosyan: Nanotech Conference \& Expo June 18-21, Santa Clara, California, (2012).

[5] Y. J. Peng, Y. Q. Liu, Y. H. Wang,S. P. Zhang, and Y. Q. Yang: Acta Physics Sinica, 58, (2009) 0655

[6] M. A. Zamkov, R. W. Conner, and D. D. Dlott: J. Phys. Chem. C, 111, (2007) 10278.

[7] W. K. Lewis, C. G. Rumchik, P. B. Broughton, and C. M. Lindsay: Journal of Applied Physics 111,(2012) 014903.

[8] Y. Wang, X. L. Song, W. Jiang, G. D. Deng, X. D. Guo, H. Y. Liu, F. S. Li: Tran. Nonferrous Met. Soc. China, 24,(2014) 263.

[9] P. E. Bocanegra, V. Sarou-Kanian, C. Chauveau, I. Gökalp: Third European Combustion Meeting ECM 2007.
[10]W. L. Perry, B. C. Tappan, B. L. Reardon, V. E. Sanders, and S. F. Son: Journal of Applied Physics 101, (2007) 064313.

[11]Y. Q. Yang, S. F. Wang, Z. Y. Sun, and D. D. Dlott: Journal of Applied Physics, 95, (2004) 3667.

[12]T. Q. Qiu, and C. L. Tien: J. Heat Transfer, 115, (1993) 835.

[13]M. R. Huyeh, S. Volz, and B. Palpant: Phys. Rev. B, 78, (2008) 125408.

[14]H. Ritter and S. Braun, Propellants, Explos., Pyrotech. 26, (2001) 311.

[15]R. R. Letfullin, T. F. George, G. C. Duree, and B. M. Bollinger: Advances in Optical Technologies, 2008, (2008) 251718

[16]J. G. Tian, H. H. Wang, W. Y. Zhou, T. Li, C. P. Zhang, and G. Y. Zhang: Chin. Phys. Lett., 17, (2000) 510.

[17]V. K. Pustovalov: Chem. Phys. Lett., 421, (2006) 142.

[18]H. P. Myers: "Introductory Solid State Physics" (Publisher, Londan: Taylor \& Francis, 1990) p.168.

[19]M. R. Huyeh, and B. Palpant: J. Appl. Phys., 96, (2004) 4475.

[20]J. K.Chen, D. Y. Zhou, and J. E. Beraun: International Journal of Heat and Mass Transfer, 49, (2006) 307.

[21]Y. J. Peng, Y. H. Wang, B. Palpant, X. He, X. X. Zheng, and Y. Q. Yang: International Journal of Modern Physics B, 24, (2010) 381.

[22]H. S. Dong, R. Z. Hu, P. Yao, and X. Y. Zhang: "Thermogram Set of Energetic Materials" (Publisher, National Defence Industry Press,2002), p.164.

[23]Z. B. Lin and L. V. Zhigilei: Physical Review B, 77 (2008) 075133.

[24]C. Kittel: "Introduction to solid state physics" (Publisher, New York: Wiley, 1996), p.157.

[25]J. L. Hostetler, A. N. Smith, D. M. Czajkowsky, and P. M. Norris: Appl. Opt., 38, (1999) 3614.

[26]L. D. Zhu, F. Y. Sun, and J. Zhu: Heat and Mass Transfer Conference of Chinese Society of Engineering Thermophysics, 113, (2012) p.188.

[27]H. F. Hu, Y. Ji, Y. Hu, X. Y. Ding, X. W. Liu, J. H. Guo, X. L. Wang, and H. C. Zhai: Chin. Phys. B, 20,(2011) 044204.

[28]G. V. Hartland: INt. J. Nanotechnology, 1, (2004) 307.

[29]O. L. Muskens, N. D. Fatti, and F. Vallee: Nano Letters, 6, (2006) 552.

[30]P. K. Jain, W. Qian, and M. A. El-Sayed: J. Am. CHEM. SOC. 128 (2006) 2426.

(Received: December 20, 2013, Accepted: January 15, 2015) 